\title{
Regulation of DNA Methylation on Key Parasitism Genes of Cysticercus Cellulosae Revealed by Integrative Epigenomic-Transcriptomic Analyses
}

\section{Xinrui Wang}

Inner Mongolia University For Nationalities

\section{Weiyi Song}

Inner Mongolia University for Nationalities

\section{Yining Song}

Inner Mongolia University for Nationalities

\section{Guanyu Ji}

Huahan Industrial Zone

\section{Xuenong Luo}

Key Laboratory of Veterinary Parasitology of Gansu Province

Shumin Sun ( $\nabla$ shums1975@163.com )

inner mongolia university for nationalities

\section{Research}

Keywords: Cysticercus cellulosae, DNA methylation, epigenetics, gene regulation

Posted Date: August 6th, 2020

DOI: https://doi.org/10.21203/rs.3.rs-51736/v1

License: (1) This work is licensed under a Creative Commons Attribution 4.0 International License. Read Full License

Version of Record: A version of this preprint was published at Hereditas on August 12th, 2021. See the published version at https://doi.org/10.1186/s41065-021-00195-9. 


\section{Abstract}

Background: The life cycle of Taenia solium is characterized by different stages of development, requiring various kinds of hosts that can appropriately harbor the eggs (proglottids), the oncospheres, the larvae and the adults. Similar to other metazoan pathogens, T. solium undergoes transcriptional and developmental regulation via epigenetics during its complex lifecycle and host interactions.

Result: In the present study, we integrated whole-genome bisulfite sequencing and RNA-seq technologies to characterize the genome-wide DNA methylation and its effect on transcription of Cysticercus cellulosae of $T$. solium. We confirm that the $T$. solium genome in the cysticercus stage is epigenetically modified by DNA methylation in a pattern similar to that of other invertebrate genomes, i.e., sparsely or moderately methylated. We also observed an enrichment of non-CpG methylation in defined genetic elements of the $T$. solium genome. Furthermore, an integrative analysis of both the transcriptome and the DNA methylome indicated a strong correlation between these two datasets, suggesting that gene expression might be tightly regulated by DNA methylation. Importantly, our data suggested that DNA methylation might play an important role in repressing key parasitism-related genes, including genes encoding excretion-secretion proteins, thereby raising the possibility of targeting DNA methylation processes as a useful strategy in therapeutics of cysticercosis.

\section{Introduction}

Cysticercus cellulosae, the larval stage of $T$. solium, resides in the central nervous system, skeletal muscle, and other organs of both pigs and humans (Schantz et al., 1993), resulting in the high prevalence of cysticercosis worldwide. As a neglected tropical disease prioritized by the World Health Organization, serious human disease burden (Sciutto et al., 2000) and annual economic losses in livestock are caused by infection with this pork tapeworm. To better control this disease, the mechanisms of transcriptional and developmental regulation during its complex lifecycle and host interactions should be better understood.

ADNA methylation, i.e., 5-methylcytosine (m5C) is an important epigenetic mechanism that is present in the genomes of Trichinella spiralis (Gao et al., 2012) and Platyhelminthes (Schistosoma mansoni (Geyer et al., 2011)) parasitic nematodes. Via regulating gene transcription, DNA methylation plays an important role in parasitism. Similar to T. solium, S. mansoni belongs the phylum of Platyhelminthes. A previous study showed that $S$. mansoni contains conserved DNA methyltransferase 2 (DNMT2) and methyl-CpG binding proteins (MBD) (Geyer et al., 2011). Importantly, demethylation induced by 5-azacytidine can disrupt egg production and maturation, indicating an essential role for DNA methylation in the normal development of this parasitic worms in this phylum (Geyer et al., 2011).

In the present study, we aimed to identify functional DNA methylation machinery and detect cytosine methylation levels in the cysticercus cellulosae of $T$. solium based on a draft genome that has been sequenced and annotated previously (Aguilar-Diaz et al., 2006). To achieve this aim, we applied the 
whole-genome bisulfite sequencing (WGBS) method to characterize the genome-wide DNA methylation pattern at single-base resolution (Lister \& Ecker, 2009). Based on this unbiased characterization, our results confirm that in the cysticercus stage, the T. solium genome (Aguilar-Diaz et al., 2006) is epigenetically modified by DNA methylation in a pattern similar to that of other invertebrate genomes, i.e., sparsely or moderately methylated (Feng et al., 2010; Zemach et al., 2010). We also observed an enrichment of non- $\mathrm{CpG}$ methylation in defined genetic elements of $T$. solium genome, which is a pattern different from mammalian methylomes (Feng et al., 2010; Zemach et al., 2010). Furthermore, we applied RNA-seq technology to profile gene expression. An integrative analysis on both the transcriptome and DNA methylome indicated a strong correlation between these two datasets, suggesting that gene expression might be tightly regulated by DNA methylation. Importantly, our data suggested that DNA methylation might play an important role in repressing key parasitism-related genes, including genes encoding excretion-secretion proteins. In summary, for the first time, we provide data to characterize the DNA methylome and the transcriptome of the T. solium cysticercus cellulosae. Our data will be valuable to the community and will allow researchers to provide new insights into the mechanism of methylation in cysticercosis in future studies.

\section{Materials And Methods}

\section{Ethics Statement}

Animals were treated according to the guidelines of the National Institute of Health (publication No. 8523, revised 1996). Animal protocols have been reviewed and approved by the Ethical Committee of the Jilin University affiliated with the Provincial Animal Health Committee, Jilin Province, China (Ethical Clearance number IZ-2009-08).

\section{Sample collection and nuclei acid extraction}

Individual cysticerci were isolated from a single, naturally infected Landrace (Inner mongolia, China) and rinsed thoroughly several times with phosphate-buffered saline. The cysticerci were first frozen in liquid nitrogen and then finely ground to a powder-like texture. Genomic DNA was extracted using the phenol chloroform extraction method, and total RNA was purified using Trizol reagent (Invitrogen, CA, USA) according to the manufacturer's instructions. RNA was dissolved in diethylpyrocarbonate (DEPC)-treated water and treated with DNase $\mathbb{~ ( I n v i t r o g e n , ~ C A , ~ U S A ) . ~ T h e ~ q u a n t i t y ~ a n d ~ q u a l i t y ~ o f ~ t h e ~ D N A ~ a n d ~ R N A ~ w e r e ~}$ tested by ultraviolet-Vis spectrophotometry with a NanoDrop 2000 (Thermo Scientific CA, USA).

\section{BlastP searches and phylogenetic analysis of DNMTs}

Reciprocal BlastP comparisons were first performed to identify DNMTs and MBD orthologs. Significant hits were defined as those satisfying the following criteria: E-value $<1$ e- 5 and aligned segments covering at least $30 \%$ of the sequence length of the hit. For phylogenetic analysis, multiple sequence alignment 
was performed by Clustal W (Saitou \& Nei, 1987). The MEGA7 with the neighbor-joining method (Jones et al., 1992; Kumar et al., 2016) based on the JTT+ G (Jones-Taylor-Thornton and Gamma Distribution) model was applied to reconstruct the phylogenetic tree.

\section{MethylC-seq library construction and sequencing}

Prior to library construction, $5 \mu \mathrm{g}$ of genomic DNA extracted from a cysticercosis body was spiked with 25 ng unmethylated lambda DNA (Promega, Madison, WI, USA) and fragmented using a Covarias sonication system to a mean size of approximately $200 \mathrm{bp}$. After fragmentation, libraries were constructed according to the Illumina Paired-End protocol with some modifications. Briefly, purified randomly fragmented DNA was treated with a mix of T4 DNA polymerase, Klenow fragment and T4 polynucleotide kinase to repair blunt ends and phosphorylate the ends. The blunt DNA fragments were subsequently 3' adenylated using Klenow fragment ( 3 '-5' exo-), followed by ligation to adaptors synthesized with 5'methylcytosine instead of cytosine using T4 DNA ligase. After each step, DNA was purified using a QIAquick PCR purification kit (Qiagen, Shanghai, China). Next, a ZYMO EZ DNA Methylation-Gold Kit^ ${ }^{\top M}$ (ZYMO Research, Irvine, CA, USA) was employed to convert unmethylated cytosine to uracil, according to the manufacturer's instructions, and 220 to 250 bp converted products were size selected. Finally, PCR was carried out in a final reaction volume of $50 \mu \mathrm{l}$ consisting of $20 \mu \mathrm{l}$ of size selected fractions, $4 \mu \mathrm{l}$ of 2.5 mM dNTPs, $5 \mu$ l of $10 \times$ buffer, $0.5 \mu$ l of JumpStart ${ }^{\text {TM }}$ Taq DNA Polymerase, $2 \mu \mathrm{l}$ of PCR primers and 18.5 $\mu$ l water. The thermal cycling program was $94^{\circ} \mathrm{C}$ for 1 minute; 10 cycles of $94^{\circ} \mathrm{C}$ for $10 \mathrm{~s}, 62^{\circ} \mathrm{C}$ for $30 \mathrm{~s}$, $72^{\circ} \mathrm{C}$ for $30 \mathrm{~s}$; and then a 5 -minute incubation at $72^{\circ} \mathrm{C}$ before holding the products at $12^{\circ} \mathrm{C}$. The PCR products were purified using a QIAquick gel extraction kit (Qiagen). Before analysis with an Illumina Hiseq2500, the purified products were analyzed using a Bioanalyzer analysis system (Agilent, Santa Clara, CA, USA) and quantified by real-time PCR. Raw sequencing data were processed using the lllumina base-calling pipeline (Illumina Pipeline version 1.3.1). The sodium bisulfite non-conversion rate was calculated as the percentage of cytosines sequenced at cytosine reference positions in the lambda genome.

\section{RNA-seq library construction and sequencing}

Total RNA was extracted using the Invitrogen TRIzol Reagent and then treated with RNase-free DNase I (Ambion, Guangzhou, China) for 30 minutes. The integrity of total RNA was checked using an Agilent 2100 Bioanalyzer. cDNA libraries were prepared according to the manufacturer's instructions (Illumina). The poly(A)-containing mRNA molecules were purified using Oligo (dT) Beads (Illumina) and $20 \mu \mathrm{g}$ of total RNA from each sample. Tris $\mathrm{HCl}(10 \mathrm{mM})$ was used to elute the mRNA from the magnetic beads. To avoid priming bias when synthesizing the CDNA, mRNA was fragmented before cDNA synthesis. Fragmentation was performed using divalent cations at an elevated temperature. The cleaved mRNA fragments were converted into double-stranded cDNA using SuperScript II, RNase H and DNA Pol I, primed by random primers. The resulting cDNA was purified using a QIAquick PCR Purification Kit 
(Qiagen). Then, the cDNA was subjected to end repair and phosphorylation using T4 DNA polymerase, Klenow DNA polymerase and T4 Polynucleotide Kinase (PNK). Subsequent purifications were performed using the QIAquick PCR Purification Kit (Qiagen). These repaired cDNA fragments were 3'-adenylated using KlenowExo (Illumina) and purified using the MinElute PCR Purification Kit (Qiagen), producing cDNA fragments with a single ' $A$ ' base overhang at the 3 ' end for subsequent ligation to the adapters. Illumina PE adapters were ligated to the ends of these 3 '-adenylated cDNA fragments and then purified using the MinElute PCR Purification Kit (Qiagen). To select a size range of templates for downstream enrichment, the products of the ligation reaction were purified on $2 \%$ TAE-Certified Low-Range Ultra Agarose (Bio-Rad, Hercules, CA, USA). cDNA fragments (200 $\pm 20 \mathrm{bp}$ ) were excised from the gel and extracted using the QIAquick Gel Extraction Kit (Qiagen). Fifteen rounds of PCR amplification were performed to enrich the adapter-modified cDNA library using primers complementary to the ends of the adapters (PCR Primer PE 1.0 and PCR Primer PE 2.0; Illumina).

\section{Transcriptome mapping}

RNA-seq reads were trimmed to a maximum length of $80 \mathrm{bp}$, and stretches of bases having a quality score $<30$ at the ends of the reads were removed. Reads were mapped using Tophat 2.0.11 (Trapnell et al., 2009). As reference sequence for the transcriptome mapping we used the current assembly of the $T$. solium database (Pacheco et al., 2005). Expression was quantified using cufflinks 2.1.1 (Trapnell et al., 2010). RepeatMasker (Tarailo-Graovac \& Chen, 2009) were used to identify tandem repeats.

\section{Bisulfite mapping and methylation calling}

Reads were trimmed to a maximal length of $125 \mathrm{bp}$, and stretches of bases having a quality score $<30$ at the ends of the reads were removed. Reads were mapped using BSMAP 2.2.74 (Xi \& Li, 2009). As a reference sequence for the bisulfite mapping we used the current assembly of the $T$. solium genome (Pacheco et al., 2005). Only reads mapping with both partners of the read pairs at the correct distance were used. The CpG-specificity was calculated by determining the number of cytosines called in all mapped reads at all non-CpG positions and dividing by the number of all bases in all mapped reads at all non-CpG positions. Methylation ratios were determined using a Python script (methratio.py) distributed together with the BSMAP package for both the forward and reverse strands.

\section{Protein network analyses}

The STRING online tool (Fonseca et al., 2006) was used with default parameters. Peptide sequences of key genes were the input and were aligned to Caenorhabditis elegans protein sequences.

\section{Data availability}


The $T$. solium methylome data have been deposited at NCBI/GEO/ under the accession number GSE84086.

\section{Results}

\section{The presence of DNA methylation in the T. solium genome}

The methylation status of DNA is related to three types of enzymes, including DNA methyltransferases, which affect maintenance methylation and de novo methylation. To understand whether T. solium possesses the ability to methylate DNA, we first conducted a reciprocal Blast alignment to identify genes that might be homologous to known DNA (cytosine-5)-methyltransferases. As a result, two genes (Scaffold00200.gene8095 and LongOrf.asmbl_16366) were identified that are homologous to DNMT3B and $D N M T 2$, respectively, with high sequence similarity (e-value $<1 \mathrm{e}-10$ ). Scaffold00067.gene4890 was aligned (e-value $<1 \mathrm{e}-5$ ) with either DNMT3A or DNMT3B from multiple species. In addition, more than one gene was matched with DNMT1, among which Scaffold00068.gene4920 had the best hit (e-value $<1 \mathrm{e}$ 10) (Table S1). Phylogenetic analyses by MEGA7 also supported these results (Figure S1). Moreover, we searched for genes homologous to methyl-CpG binding domain protein (MBD). Two candidate genes (LongOrf.asmbl_5021 and LongOrf.asmbl_14047) were homologous to MBDs in multiple species, including Echinococcus granulosus, which is closely related to T. solium evolutionarily (Table S1 and Figure S2). A full repertoire of functionally conserved amino acid residues was identified for both the potential DNMT2 and DNMT3 and the MBDs of T. solium, indicating that these proteins are functionally active (Table S2). However, a high level of divergence between $T$. solium and other species was observed for DNMT1 homologs (Table S2), which was in agreement with previous studies.

Given these results, we assessed the genome-wide DNA methylation profiles in $T$. solium using MethylCSeq. There were 54.21 million raw reads generated (Table S3). BSMAP (Xi \& Li, 2009) was used to align the sequenced reads to the $T$. solium reference sequence, reaching an approximately $76.41 \%$ mapping rate. The average read depth was 11.32 per strand, while on average, over $50 \mathrm{Mb}(90.52 \%)$ of each strand of the T. solium reference sequence was covered. Because of the potential occurrence of non-conversion and thymidine-cytosine sequencing errors, the false-positive rate was estimated by calculating the methylation level of lambda DNA, which is normally unmethylated (Materials and methods). We then applied the error rate $(0.0041)$ to correct methylated cytosine sites $(\mathrm{mC})$ identification according to the method described by Lister et al. (Lister \& Ecker, 2009), which is based on a binomial test and false discovery rate constraints. As a result, approximately 76.6 thousand $\mathrm{mCs}$ were estimated in the $T$. solium genome (accounting for $0.20 \%$ of the total cytosines sequenced with depth $\geq 5 \mathrm{X}$ ). Both symmetrical CpG methylation and asymmetrical non- $\mathrm{CpG}$ methylation were revealed.

\section{Characterization of overall methylation patterns}


We further characterized the global patterns of DNA methylation in the genomes of $T$. solium. First, we showed the percentage of methylated cytosine of each sequence context. Among the 76.6 thousand mCs across the entire genome, a majority (69.5\%) were in the context of $\mathrm{CHH}$. In contrast, only $15.38 \%$ and $15.12 \%$ of the $\mathrm{mCs}$ were located in the contexts of $\mathrm{CHG}$ and $\mathrm{CpG}$, respectively (Figure S3 A). Furthermore, most of the $\mathrm{CpG}$ and non-CpGs displayed a low methylation fraction ( $<30 \%)$ (Figure S3B and C). These patterns are highly different from mammalian methylomes, in which most $5 \mathrm{mCs}$ are located in CpG contexts and the majority of the CpGs are highly methylated (>50\%) (Feng et al., 2010). Since most (69.5\%) of the $\mathrm{mCs}$ in the $\mathrm{T}$. solium genome were in the $\mathrm{CHH}$ context, we further analyzed the sequence context of $\mathrm{mCHHs}$ across the entire genome to further examine whether there is any sequence bias in the enrichment of cytosine methylation in the $\mathrm{CHH}$ context. As a result, mCpA was shown to be preferentially enriched within the methylated $\mathrm{CHH}$ dinucleotide (Figure 1A). There were more than 21,000 methylated CpAs in each strand, meaning that $55.73 \%$ of total CpAs were methylated in the entire genome (Figure 1B, D). This result was consistent with reports that $\mathrm{mCpA}$ was predominantly found in another tapeworm, $S$. mansoni (Weber et al., 2007; Zhang, 2008). With regard to methylation levels, we did not observe significant differences among different sequence contexts for mCs (Figure 1C).

We next examined whether there was any preference for the distance between adjacent sites of DNA methylation in the T. solium genome. The relative distance between $\mathrm{mCs}$ in each context within 50 nucleotides in introns was then analyzed because of the steady methylation without any selective pressure by protein coding genes in intron regions. Similar to the periodicity of 8-10 bases revealed in previous studies on the Arabidopsis and human genomes (Cokus et al., 2008), we also observed a strong tendency of peaked enrichment of mCpA sites, which might be explained by a single turn of the DNA helix (Figure 1E). Moreover, we found that $\mathrm{mCpT}$ revealed a similar periodicity of 8-12 bases (Figure 1F), though the numbers of cytosines in the context of $\mathrm{CpG}$ and $\mathrm{CpC}$ were too few to yield reliable results (Figure S3D and E). In summary, our results indicated that the molecular mechanisms governing de novo methylation at $\mathrm{CPA}$ sites may be similar among the cysticercus and the plant and animal kingdoms.

We then examined the distribution of methylation levels for the four categories of methylated cytosines across the entire genome. In general, similar mosaic distribution patterns were observed for methylation levels of all types of $\mathrm{mCs}$, that is, relatively highly methylated domains were interspersed within regions with low methylation (Figure S4A). Furthermore, the distribution of $\mathrm{mCs}$ across the genome was also uneven; dense $\mathrm{mCs}$ of specific categories were occasionally enriched in specific scaffolds (Figure S4B). Such a pattern has been observed in previous studies on other invertebrates. We also examined the patterns of methylation in annotated elements, including genes, tandem repeats, and transposable elements. The methylation percentage of each cytosine context in exons was higher than that in other annotated elements, especially CpAs, which accounted for a more than 2-fold greater percentage than the other contexts in exons (Figure 2A).

We then examined the average methylation level in each element, which showed that average CpG methylation levels were higher than those other types of methylated cytosines, similar to mammalian genomes. However, the genome-wide pattern was again divergent from mammalian genomes, as higher 
average methylation in exons and lower methylation in introns of CpG sites were observed (Figure 2B, C and Figure S5). The trend for the average methylation of $\mathrm{CpC}$ and $\mathrm{CpT}$ was similar to that of $\mathrm{CpG}$. However, a uniform distribution of $\mathrm{CpA}$ methylation levels in each annotated element was displayed (Figure $2 \mathrm{C}$ and Figure S5). We also analyzed the methylation level of each cytosine context in repeat regions (Figure 2D, E). Previous studies have indicated that transposable elements are usually unmethylated in the honey bee Apis mellifera and silkworm Bombyx mori (Lyko et al., 2010; Xiang et al., 2010). In cysticercus, we observed a similar phenomenon as the above species except that relatively highly methylated rRNAs were observed in T. solium. Notably, CpAs were methylated at a higher level or frequency than other types in LINE/L1 (Figure 2D, E).

\section{The relationship between methylation and gene expression}

It was reported that DNA methylation plays an important role in regulating gene expression. We evaluated gene expression in T. solium using Illumina high-throughput RNA-seq technology. Most of the raw reads could be uniquely mapped to previously annotated genes (88.17\%). A total of 9,718 annotated genes out of 11,903 could be aligned with at least one unique read. To characterize the relationship between DNA methylation and gene expression, we divided the expressed genes with at least one read into quartiles of expression levels. We then examined the distribution of methylation levels for different quartiles of expressed genes and genes exhibiting no expression. High $\mathrm{CpG}$ and $\mathrm{CpC}$ methylation levels were observed in upstream and exon regions of genes with the lowest expression. Moreover, a negative correlation could also be observed between $\mathrm{CpA}$ and $\mathrm{CpT}$ methylation levels of upstream and exons and expression levels of these expressed genes. However, for silent genes, mainly high $\mathrm{CpG}$ and $\mathrm{CpC}$ methylation levels of downstream regions were observed (Figure 3). Taken together, methylation levels of $\mathrm{mCs}$ from both $\mathrm{CpG}$ or non- $\mathrm{CpG}$ sequence contexts were correlated with gene expression levels, though different regulation mechanisms might be involved.

Next, to infer whether methylated genes were enriched for specific molecular functions, we filtered out a total of 1,647 of the genes with the lowest expression and 1,354 of the most highly expressed genes, based on the criteria that at least one $\mathrm{mC}$ was present within their genic regions. Then, we applied the WEGO (Web Gene Ontology Annotation Plotting) tool (Ye et al., 2006) to functionally categorize the gene ontology (GO) terms of these genes. We found that these two sets of genes displayed similar patterns of GO enrichment, specifically, "cell" and "cell part" in Cellular Component, "binding" and "catalytic" Molecular Functions, and "cellular process" and "metabolic process" in Biological Process were relatively enriched. This result suggested that the genes heavily regulated by DNA methylation were more prone to signaling regulation or interaction with environmental factors, e.g., diet or metabolism (Figure S6). In summary, these results suggested the potential for the regulation of $T$. solium genes by DNA methylation, especially those that function as regulators of cell-cell or cell-environmental communication. Furthermore, different molecular mechanisms might be involved depending on different $\mathrm{mC}$ contexts and genes. 


\section{Regulation of DNA methylation on key parasitism genes of T. solium}

To obtain further insight into the epigenetic regulation of parasite development, survival and parasite-host interactions of $T$. solium, we next studied conserved genes across tapeworm-species and genes encoding excretion-secretion proteins (ESPs) in T. solium. For conserved genes, we applied a gene set that was reported previously in a study by Bjorn Victor et al., in which 261 genes conserved between Taenia and Echinococcus tapeworms were obtained by comparing the transcriptomes of five important intestinal parasites, including T. multiceps, T. solium, E. granulosus, E. multilocularis and T. pisiformis (Robert McMaster et al., 2016). Based on their results, we further retrieved 216 genes with the best blastx hit for each contig (e<1e-10) and studied their DNA methylation status. A total of 190 of these genes contained at least one $\mathrm{mC}$ across their genic regions. As indicated in Figure $4 \mathrm{C}, \mathrm{CpG}$ and $\mathrm{CpC}$ methylation levels in upstream and exon regions were higher than other types of methylation and in other genic regions. $\mathrm{A}$ further examination of the 190 genes revealed that 71 genes contained $\mathrm{CpG}$ or $\mathrm{CpC}$ methylation within their upstream or exon regions. Therefore, we searched for extensively methylated genes based on the criterion that the $\mathrm{CpG}$ and $\mathrm{CpC}$ methylation levels of the examined gene were significantly higher than the average value of the 71 genes. As a result, we revealed 14 conserved genes that were extensively methylated on $\mathrm{CpG} / \mathrm{CpC}$ sites within their upstream regions and exons $(\mathrm{p}<0.05)$. Compared with those 26 genes without $\mathrm{mC}$, we found these 14 genes were expressed at a significantly lower level (Figure 4A), suggesting DNA methylation is a key mechanism for the transcriptional regulation of these conserved genes. For ESPs, we also applied a dataset containing 76 ESPs for T. solium, which was identified by Bjorn Victor et al. using a proteomics strategy (Victor et al., 2012). We applied the BlastP algorithm to align these ESPs back to the genome and revealed 111 gene sequences that might encode these ESPs (Table S4). Using the same criterion for conserved genes, we found 13 extensively methylated genes. Similarly, gene expression comparisons again revealed that these 13 genes were expressed at significantly lower levels than the 26 non-methylated genes (Figure 4A). Using a similar strategy, we also looked into genes containing methylated $\mathrm{CpAs}$ and $\mathrm{CpTs}$ within their upstream or exon regions. However, no clear difference in gene expression levels was observed (Figure 4B). These results indicated that $\mathrm{CpG} / \mathrm{CpC}$ methylation in upstream regions and exons played a major role in $T$. solium gene repression. Furthermore, we found a different distribution pattern between $\mathrm{mCpG}$ and $\mathrm{mCpC}$ for these repressed genes, in which $\mathrm{mCpG}$ were mostly distributed in upstream regions, while $\mathrm{mCpC}$ were more often in exons (Figure S5). Based on the above analyses, we revealed 27 key genes that might be repressed by DNA methylation mechanisms. Interestingly, a protein-protein interaction analysis using the STRING online tool (Fonseca et al., 2006) indicated strong mutual interactions among these conserved proteins and ESPs (Figure 5) based on annotation of the model organism Caenorhabditis elegans.

\section{Discussion}

The larval stage of the pork tapeworm T. solium is responsible for cysticercosis, which represents an important public health problem that occurs mainly in developing countries. T. solium cysticerci have 
developed diverse mechanisms to protect themselves from host immune attack (Hewitson et al., 2009), among which epigenetics may play an important role in gene regulation related to parasitism (Robert McMaster et al., 2016). Recently, Geyer et al. found that essential DNA methylation machinery components, such as DNMT2 and MBD, are well conserved throughout the Platyhelminthes (Geyer et al., 2013; Geyer et al., 2011). Invertebrate DNMT2s are believed to retain strong DNA methyltransferase activity (Phalke et al., 2009), which is different from vertebrate DNMT2s, which are considered tRNA methyltransferases (Goll et al., 2006). Our computational searches indicated that both DNMT2 and DNMT3 are found in T. solium, which implies the potential existence of a more sophisticated DNA methylation machinery. In addition, MBD2/3 homologs were also identified in the $T$. solium genome.

Based on these results, our present study focused on characterizing the DNA methylome and transcriptome of $T$. solium cysticerci, aiming for providing comprehensive omics profiles for this important parasitic stage of $T$. solium. We revealed a mosaic methylation pattern in $T$. solium that is typical of other invertebrates (Bird et al., 1979; del Gaudio et al., 1997; Gao et al., 2012; Geyer et al., 2011; Lyko et al., 2010; Xiang et al., 2010). Cytosine methylation was predominantly found in the CpA dinucleotide context, similar to other invertebrate species, including Drosophila melanogaster (Lyko et al., 2000) and other platyhelminths such as S. mansoni (Geyer et al., 2013), which might be mediated by MBD2/3 proteins (Marhold et al., 2004; Raddatz et al., 2013). These patterns in the DNA methylome might be closely related to the activity of different DNMTs. As DNMT1 functions as a maintenance methylase by copying methylation after DNA replication with the help of Uhrf1 (Law \& Jacobsen, 2010), a lack of DNMT1 might help to explain why much non-symmetrical methylation was observed in the platyhelminth genome. We also found that a periodicity for two pairs of $\mathrm{mCpA}$ and $\mathrm{mCpT}$ sites spaced with 13 bases between the pairs, corresponding to a single turn of the DNA helix, as previously observed. A structural study of the mammalian de novo methyltransferase DNMT3A and its partner protein DNMT3L found that two copies of each form a heterotetramer that contains two active sites separated by a length of 8-10 nucleotides in a DNA helix (Huff \& Zilberman, 2014; Jia et al., 2007). Because we could not locate $D N M T 3 L$ in the $T$. solium genome, the consistent 8-10 nucleotide spacing we observed in the T. solium genome might be due to DNMT3A alone or an unknown factor other than DNMT3L.

Gene methylation is believed to be an evolutionarily ancient means of transcriptional control. Among plants, vertebrates and some invertebrates such as T. spiralis, the notion that methylation in promoters primarily represses genes by impeding transcriptional initiation has been widely accepted (Weber et al., 2007; Zemach et al., 2010; Zhang, 2008), whereas intermediate levels of expression have been associated with genes experiencing the greatest extent of methylation in the gene body, indicating a bell-shaped relationship (Jjingo et al., 2012; Nanty et al., 2011; Zilberman et al., 2007). However, in invertebrates, such as the fungus Neurospora crassa (Rountree \& Selker, 1997) and the silkworm Bombyx mori, transcription initiation is unaffected. Thus, DNA methylation shows remarkable diversity in its extent and function across eukaryotic evolution. In our T. solium results, we also found that methylation levels of mCs were correlated with gene expression levels. Depending on different sequence contexts, methylation seemed to function differently in transcriptional regulation. Intriguingly, high $\mathrm{CpG}$ and $\mathrm{CpC}$ methylation levels of downstream regions, but not of promoter regions, were observed for silent genes (Figure 4). In contrast, 
upstream methylation seemed to mostly affect genes with low expression. Currently, knowledge on methylation patterns and their effects on gene regulation in non-vertebrates are still limited, though species-specific diversity has been observed (Schubeler, 2015). Therefore, more data should be collected for the DNA methylomes of each specific species to characterize their patterns and functions.

In addition to characterizing the general distribution pattern of genome-wide DNA methylation, we also focused on methylation status of important $T$. solium genes. Based on previous studies, we looked into 27 extensively methylated genes that are important for $T$. solium development, survival and parasite-host interactions. We found 13 of these 27 genes mutually interacted based on annotations in the model organism C. elegans. Specifically, ESPs formed the core of the protein-protein-interaction network, while proteins encoded by conserved genes directly interacted with specific ESPs. Among the ESPs that were potentially regulated by DNA methylation, we found that two heat shock proteins (HSPs), hsp-90 and hsp1 , were highlighted and mutually interacted. The heat shock response is a general homeostatic mechanism that protects cells and organisms from the deleterious effects of environmental stress (Ferrer et al., 2005). Together with COX-2, these proteins were previously reported to be important parasitismrelated proteins (Choi \& Chu, 2012). Furthermore, we also revealed two genes encoding diagnostic antigens that might be regulated by DNA methylation, including diagnostic antigen gp50 and an $8 \mathrm{kDa}$ diagnostic protein. GP50 is a glycosylated and GPI-anchored membrane protein. In recent years, one component of the lentil lectin purified glycoprotein (LLGP) antigens has been used for antibody-based diagnosis of cysticercosis (Hancock et al., 2004). The $8 \mathrm{kDa}$ family members are metacestode excretory/secretory glycoproteins, which invoke strong antibody reactions in infected individuals (Ferrer et al., 2012). Importantly, our data suggest that DNA methylation might play a key role in repressing their transcription, implying a potential for drug development in the future that can target epigenetic modification machinery to control this important neglected tropical disease.

\section{Abbreviations}

WGBS: whole-genome bisulfite sequencing

DNMT: DNA methyltransferase

MBD: methyl-CpG binding proteins

\section{Declarations}

\section{Conflict of interest}

The authors declare that they have no competing interests.

\section{Ethics approval and consent to participate}




\section{Consent for publication}

Not applicable.

\section{Financial support}

This study was supported by the National Key Research and Development Program of China (2017YFD0501303, 2017YFC1601202, 2016YFD0500707); the National Natural Science Foundation of China (NSFC 31960707, 31160504, 31460658, 31520103916, 31872467); the Inner Mongolia Provincial Natural Science Foundation (2017MS0321).

\section{References}

1. Aguilar-Diaz, H., Bobes, R.J., Carrero, J.C., Camacho-Carranza, R., Cervantes, C., Cevallos, M.A., Davila, G., Rodriguez-Dorantes, M., Escobedo, G., Fernandez, J.L., Fragoso, G., Gaytan, P., Garciarubio, A., Gonzalez, V.M., Gonzalez, L., Jose, M.V., Jimenez, L., Laclette, J.P., Landa, A., Larralde, C., MoralesMontor, J., Morett, E., Ostoa-Saloma, P., Sciutto, E., Santamaria, R.I., Soberon, X., de la Torre, P., Valdes, V. \& Yanez, J. (2006) The genome project of Taenia solium. Parasitol Int, 55 Suppl, S127-130. doi: 10.1016/j.parint.2005.11.020.

2. Bird, A.P., Taggart, M.H. \& Smith, B.A. (1979) Methylated and unmethylated DNA compartments in the sea urchin genome. Cell, 17, 889-901.

3. Choi, W. \& Chu, J. (2012) The characteristics of the expression of heat shock proteins and COX-2 in the liver of hamsters infected with Clonorchis sinensis, and the change of endocrine hormones and cytokines. Folia Parasitol (Praha), 59, 255-263.

4. Cokus, S.J., Feng, S., Zhang, X., Chen, Z., Merriman, B., Haudenschild, C.D., Pradhan, S., Nelson, S.F., Pellegrini, M. \& Jacobsen, S.E. (2008) Shotgun bisulphite sequencing of the Arabidopsis genome reveals DNA methylation patterning. Nature, 452, 215-219. doi: 10.1038/nature06745.

5. Del Gaudio, R., Di Giaimo, R. \& Geraci, G. (1997) Genome methylation of the marine annelid worm Chaetopterus variopedatus: methylation of a $\mathrm{CpG}$ in an expressed $\mathrm{H} 1$ histone gene. FEBS Lett, 417, 48-52.

6. Feng, S., Cokus, S.J., Zhang, X., Chen, P.Y., Bostick, M., Goll, M.G., Hetzel, J., Jain, J., Strauss, S.H., Halpern, M.E., Ukomadu, C., Sadler, K.C., Pradhan, S., Pellegrini, M. \& Jacobsen, S.E. (2010) Conservation and divergence of methylation patterning in plants and animals. Proc Natl Acad Sci U S A, 107, 8689-8694. doi: 10.1073/pnas.1002720107.

7. Ferrer, E., Gonzalez, L.M., Foster-Cuevas, M., Cortez, M.M., Davila, I., Rodriguez, M., Sciutto, E., Harrison, L.J., Parkhouse, R.M. \& Garate, T. (2005) Taenia solium: characterization of a small heat 
shock protein (Tsol-sHSP35.6) and its possible relevance to the diagnosis and pathogenesis of neurocysticercosis. Exp Parasitol, 110, 1-11.

8. Ferrer, E., Sanchez, J., Milano, A., Alvarez, S., La Rosa, R., Lares, M., Gonzalez, L.M., Cortez, M.M., Davila, I., Harrison, L.J., Parkhouse, R.M. \& Garate, T. (2012) Diagnostic epitope variability within Taenia solium $8 \mathrm{kDa}$ antigen family: implications for cysticercosis immunodetection. Exp Parasitol, 130, 78-85. doi: 10.1016/j.exppara.2011.10.010.

9. Fonseca, C.T., Pacifico, L.G., Barsante, M.M., Rassi, T., Cassali, G.D. \& Oliveira, S.C. (2006) Coadministration of plasmid expressing IL-12 with 14-kDa Schistosoma mansoni fatty acid-binding protein cDNA alters immune response profiles and fails to enhance protection induced by Sm14 DNA vaccine alone. Microbes Infect, 8, 2509-2516. doi: 10.1016/j.micinf.2006.06.008.

10. Gao, F., Liu, X., Wu, X.P., Wang, X.L., Gong, D., Lu, H., Xia, Y., Song, Y., Wang, J., Du, J., Liu, S., Han, X., Tang, Y., Yang, H., Jin, Q., Zhang, X. \& Liu, M. (2012) Differential DNA methylation in discrete developmental stages of the parasitic nematode Trichinella spiralis. Genome Biol, 13, R100. doi: 10.1186/gb-2012-13-10-r100.

11. Geyer, K.K., Chalmers, I.W., Mackintosh, N., Hirst, J.E., Geoghegan, R., Badets, M., Brophy, P.M., Brehm, K. \& Hoffmann, K.F. (2013) Cytosine methylation is a conserved epigenetic feature found throughout the phylum Platyhelminthes. BMC Genomics, 14, 462. doi: 10.1186/1471-2164-14-462.

12. Geyer, K.K., Rodriguez Lopez, C.M., Chalmers, I.W., Munshi, S.E., Truscott, M., Heald, J., Wilkinson, M.J. \& Hoffmann, K.F. (2011) Cytosine methylation regulates oviposition in the pathogenic blood fluke Schistosoma mansoni. Nat Commun, 2, 424. doi: 10.1038/ncomms1433.

13. Goll, M.G., Kirpekar, F., Maggert, K.A., Yoder, J.A., Hsieh, C.L., Zhang, X., Golic, K.G., Jacobsen, S.E. \& Bestor, T.H. (2006) Methylation of tRNAAsp by the DNA methyltransferase homolog Dnmt2. Science, 311, 395-398. doi: 10.1126/science.1120976.

14. Hancock, K., Pattabhi, S., Greene, R.M., Yushak, M.L., Williams, F., Khan, A., Priest, J.W., Levine, M.Z. \& Tsang, V.C. (2004) Characterization and cloning of GP50, a Taenia solium antigen diagnostic for cysticercosis. Mol Biochem Parasitol, 133, 115-124.

15. Hewitson, J.P., Grainger, J.R. \& Maizels, R.M. (2009) Helminth immunoregulation: the role of parasite secreted proteins in modulating host immunity. Mol Biochem Parasitol, 167, 1-11. doi: 10.1016/j.molbiopara.2009.04.008.

16. Huff, J.T. \& Zilberman, D. (2014) Dnmt1-independent CG methylation contributes to nucleosome positioning in diverse eukaryotes. Cell, 156, 1286-1297. doi: 10.1016/j.cell.2014.01.029.

17. Jia, D., Jurkowska, R.Z., Zhang, X., Jeltsch, A. \& Cheng, X. (2007) Structure of Dnmt3a bound to Dnmt3L suggests a model for de novo DNA methylation. Nature, 449, 248-251. doi: 10.1038/nature06146.

18. Jjingo, D., Conley, A.B., Yi, S.V., Lunyak, V.V. \& Jordan, I.K. (2012) On the presence and role of human gene-body DNA methylation. Oncotarget, 3, 462-474. doi: 10.18632/oncotarget.497.

19. Jones, D.T., Taylor, W.R. \& Thornton, J.M. (1992) The rapid generation of mutation data matrices from protein sequences. Comput Appl Biosci, 8, 275-282. 
20. Kumar, S., Stecher, G. \& Tamura, K. (2016) MEGA7: Molecular Evolutionary Genetics Analysis Version 7.0 for Bigger Datasets. Mol Biol Evol, 33, 1870-1874. doi: 10.1093/molbev/msw054.

21. Law, J.A. \& Jacobsen, S.E. (2010) Establishing, maintaining and modifying DNA methylation patterns in plants and animals. Nat Rev Genet, 11, 204-220. doi: 10.1038/nrg2719.

22. Lister, R. \& Ecker, J.R. (2009) Finding the fifth base: genome-wide sequencing of cytosine methylation. Genome Res, 19, 959-966. doi: 10.1101/gr.083451.108.

23. Lyko, F., Foret, S., Kucharski, R., Wolf, S., Falckenhayn, C. \& Maleszka, R. (2010) The honey bee epigenomes: differential methylation of brain DNA in queens and workers. PLOS Biol, 8, e1000506. doi: 10.1371/journal.pbio.1000506.

24. Lyko, F., Ramsahoye, B.H. \& Jaenisch, R. (2000) DNA methylation in Drosophila melanogaster. Nature, 408, 538-540. doi: 10.1038/35046205.

25. Marhold, J., Kramer, K., Kremmer, E. \& Lyko, F. (2004) The Drosophila MBD2/3 protein mediates interactions between the MI-2 chromatin complex and CpT/A-methylated DNA. Development, 131, 6033-6039. doi: 10.1242/dev.01531.

26. Nanty, L., Carbajosa, G., Heap, G.A., Ratnieks, F., van Heel, D.A., Down, T.A. \& Rakyan, V.K. (2011) Comparative methylomics reveals gene-body H3K36me3 in Drosophila predicts DNA methylation and CpG landscapes in other invertebrates. Genome Res, 21, 1841-1850. doi: 10.1101/gr.121640.111.

27. Pacheco, L.G., Zucconi, E., Mati, V.L., Garcia, R.M., Miyoshi, A., Oliveira, S.C., de Melo, A.L. \& Azevedo, V. (2005) Oral administration of a live Aro attenuated Salmonella vaccine strain expressing $14-\mathrm{kDa}$ Schistosoma mansoni fatty acid-binding protein induced partial protection against experimental schistosomiasis. Acta Trop, 95, 132-142. doi: 10.1016/j.actatropica.2005.05.007.

28. Phalke, S., Nickel, O., Walluscheck, D., Hortig, F., Onorati, M.C. \& Reuter, G. (2009) Retrotransposon silencing and telomere integrity in somatic cells of Drosophila depends on the cytosine-5 methyltransferase DNMT2. Nat Genet, 41, 696-702. doi: 10.1038/ng.360.

29. Raddatz, G., Guzzardo, P.M., Olova, N., Fantappie, M.R., Rampp, M., Schaefer, M., Reik, W., Hannon, G.J. \& Lyko, F. (2013) Dnmt2-dependent methylomes lack defined DNA methylation patterns. Proc Natl Acad Sci U S A, 110, 8627-8631. doi: 10.1073/pnas.1306723110.

30. Robert McMaster, W., Morrison, C.J. \& Kobor, M.S. (2016) Epigenetics: A New Model for Intracellular Parasite-Host Cell Regulation. Trends Parasitol, 32, 515-521. doi: 10.1016/j.pt.2016.04.002.

31. Rountree, M.R. \& Selker, E.U. (1997) DNA methylation inhibits elongation but not initiation of transcription in Neurospora crassa. Genes Dev, 11, 2383-2395.

32. Saitou, N. \& Nei, M. (1987) The neighbor-joining method: a new method for reconstructing phylogenetic trees. Mol Biol Evol, 4, 406-425.

33. Schantz, P.M., Cruz, M., Sarti, E. \& Pawlowski, Z. (1993) Potential eradicability of taeniasis and cysticercosis. Bull Pan Am Health Organ, 27, 397-403.

34. Schubeler, D. (2015) Function and information content of DNA methylation. Nature, 517, 321-326. doi: 10.1038/nature14192. 
35. Sciutto, E., Fragoso, G., Fleury, A., Laclette, J.P., Sotelo, J., Aluja, A., Vargas, L. \& Larralde, C. (2000) Taenia solium disease in humans and pigs: an ancient parasitosis disease rooted in developing countries and emerging as a major health problem of global dimensions. Microbes Infect, 2, 18751890.

36. Tarailo-Graovac, M. \& Chen, N. (2009) Using RepeatMasker to identify repetitive elements in genomic sequences. Curr Protoc Bioinformatics, 25, 04.10.01-04.10.04. doi: 10.1002/0471250953.bi0410s25.

37. Trapnell, C., Pachter, L. \& Salzberg, S.L. (2009) TopHat: discovering splice junctions with RNA-Seq. Bioinformatics, 25, 1105-1111. doi: 10.1093/bioinformatics/btp120.

38. Trapnell, C., Williams, B.A., Pertea, G., Mortazavi, A., Kwan, G., van Baren, M.J., Salzberg, S.L., Wold, B.J. \& Pachter, L. (2010) Transcript assembly and quantification by RNA-Seq reveals unannotated transcripts and isoform switching during cell differentiation. Nat Biotechnol, 28, 511-515. doi: 10.1038/nbt.1621.

39. Victor, B., Kanobana, K., Gabriel, S., Polman, K., Deckers, N., Dorny, P., Deelder, A.M. \& Palmblad, M. (2012) Proteomic analysis of Taenia solium metacestode excretion-secretion proteins. Proteomics, 12, 1860-1869. doi: 10.1002/pmic.201100496.

40. Weber, M., Hellmann, I., Stadler, M.B., Ramos, L., Paabo, S., Rebhan, M. \& Schubeler, D. (2007) Distribution, silencing potential and evolutionary impact of promoter DNA methylation in the human genome. Nat Genet, 39, 457-466. doi: 10.1038/ng1990.

41. Xi, Y. \& Li, W. (2009) BSMAP: whole genome bisulfite sequence MAPping program. BMC Bioinformatics, 10, 232. doi: 10.1186/1471-2105-10-232.

42. Xiang, H., Zhu, J., Chen, Q., Dai, F., Li, X., Li, M., Zhang, H., Zhang, G., Li, D., Dong, Y., Zhao, L., Lin, Y., Cheng, D., Yu, J., Sun, J., Zhou, X., Ma, K., He, Y., Zhao, Y., Guo, S., Ye, M., Guo, G., Li, Y., Li, R., Zhang, X., Ma, L., Kristiansen, K., Guo, Q., Jiang, J., Beck, S., Xia, Q., Wang, W. \& Wang, J. (2010) Single baseresolution methylome of the silkworm reveals a sparse epigenomic map. Nat Biotechnol, 28, 516520. doi: $10.1038 /$ nbt.1626.

43. Ye, J., Fang, L., Zheng, H., Zhang, Y., Chen, J., Zhang, Z., Wang, J., Li, S., Li, R., Bolund, L. \& Wang, J. (2006) WEGO: a web tool for plotting GO annotations. Nucleic Acids Res, 34, W293-297. doi: 10.1093/nar/gkl031.

44. Zemach, A., McDaniel, I.E., Silva, P. \& Zilberman, D. (2010) Genome-wide evolutionary analysis of eukaryotic DNA methylation. Science, 328, 916-919. doi: 10.1126/science.1186366.

45. Zhang, X. (2008) The epigenetic landscape of plants. Science, 320, 489-492. doi: 10.1126/science. 1153996.

46. Zilberman, D., Gehring, M., Tran, R.K., Ballinger, T. \& Henikoff, S. (2007) Genome-wide analysis of Arabidopsis thaliana DNA methylation uncovers an interdependence between methylation and transcription. Nat Genet, 39, 61-69. doi: 10.1038/ng1929.

\section{Figures}




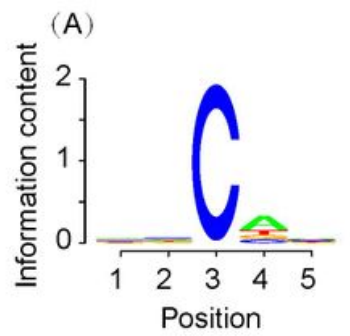

(B)

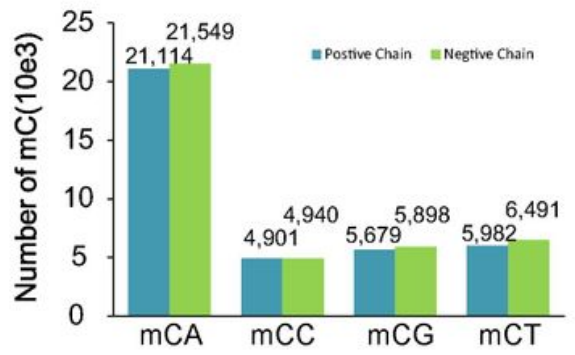

(C)
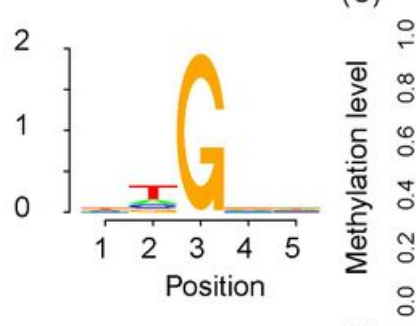

(D)

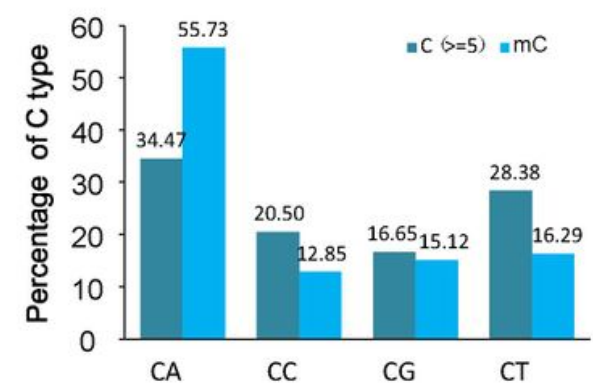

(E) 60

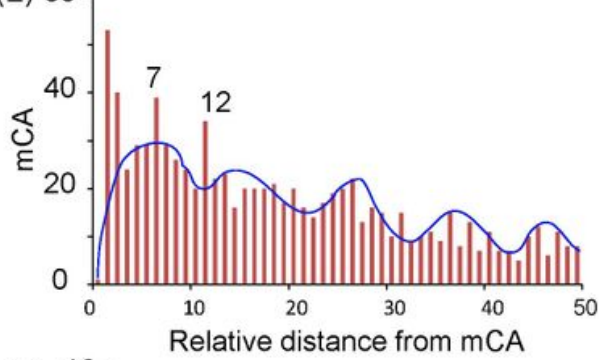

(F)

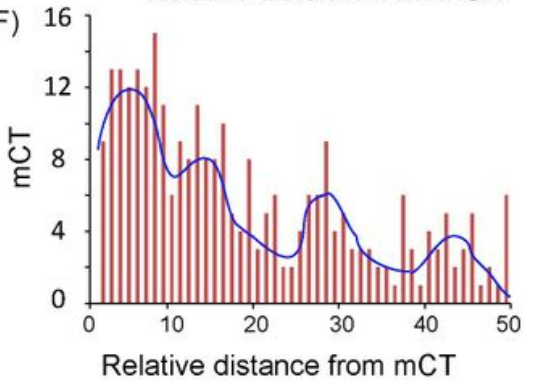

Figure 1

Cytosine DNA methylation in T. solium. (A) Logo plots of the sequences proximal to sites of cytosine DNA methylation in each sequence context in T. solium; (B) Number of mCs for each type of dinucleotide; (C) Distribution of mCs; (D) Percentage of each type of dinucleotide; $(E, F)$ Prevalence of $m C A / m C T$ sites ( $y$ axis) as a function of the number of bases between adjacent $\mathrm{mCA} / \mathrm{mCT}$ sites ( $\mathrm{x}$-axis) based on all nonredundant pair-wise distances up to $50 \mathrm{nt}$ in all introns. The blue line represents smoothing with cubic splines. 
(A)

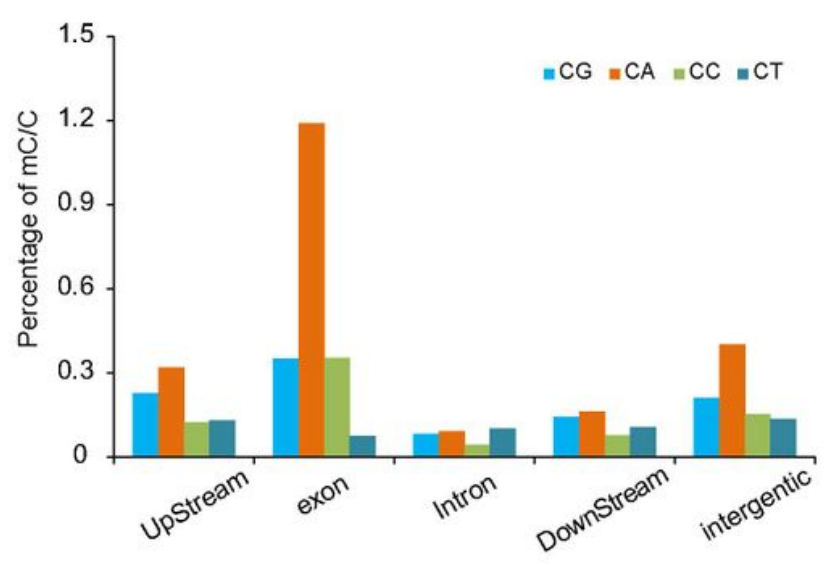

(B)

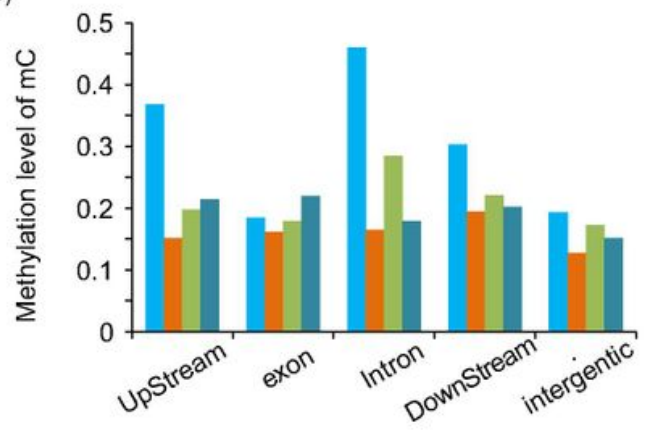

(D)
(C)
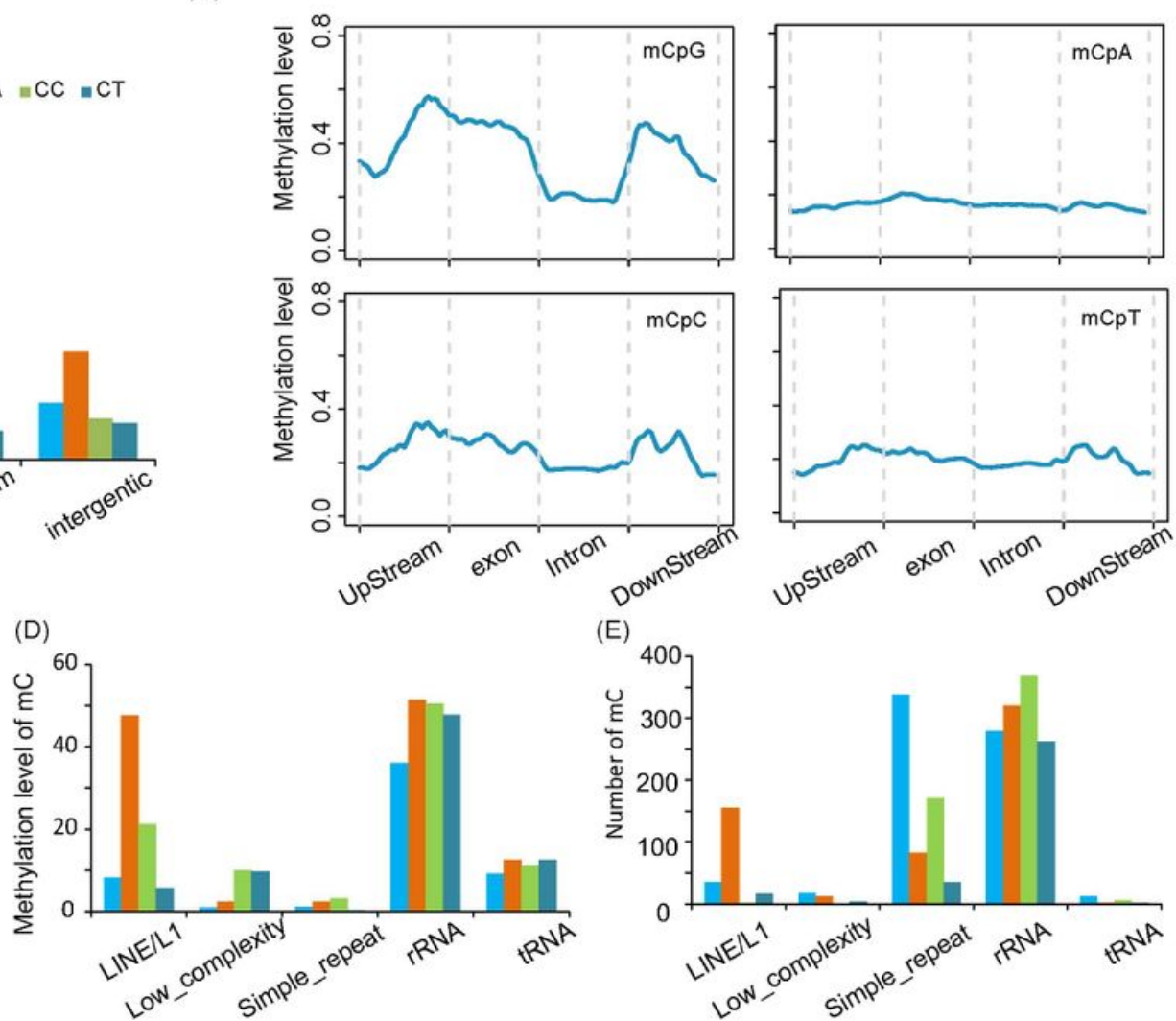

Figure 2

Average methylation levels of different genomic regions. (A, B) Average density of methylation; (C) Average density of $\mathrm{mC}$ methylation distributed on the genome. Two-kilobase regions upstream and downstream of each gene were divided into 100-bp (bp) intervals. Each coding sequence or intron was divided into 20 intervals (5\% per interval). (D) Average $\mathrm{mC}$ methylation level on repeat elements; (E) Number of $\mathrm{mCs}$ on each repeat element. 

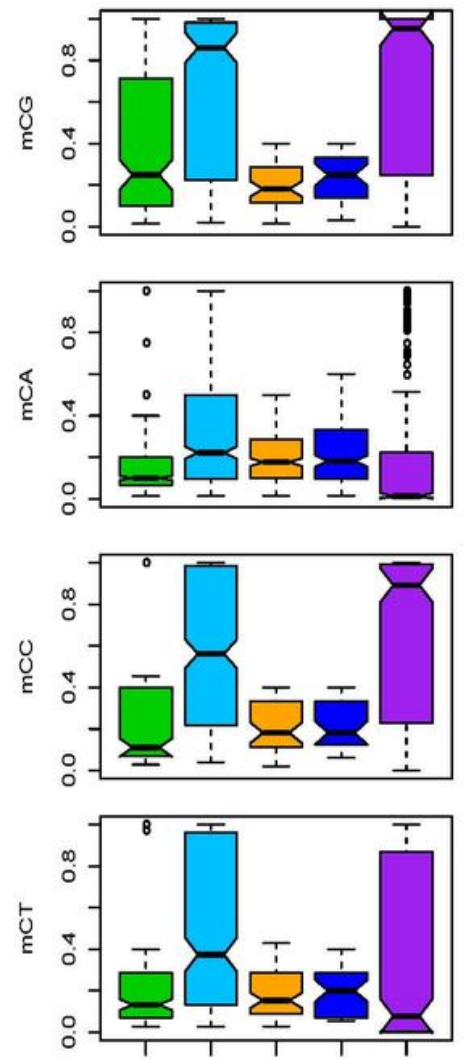

UpStream
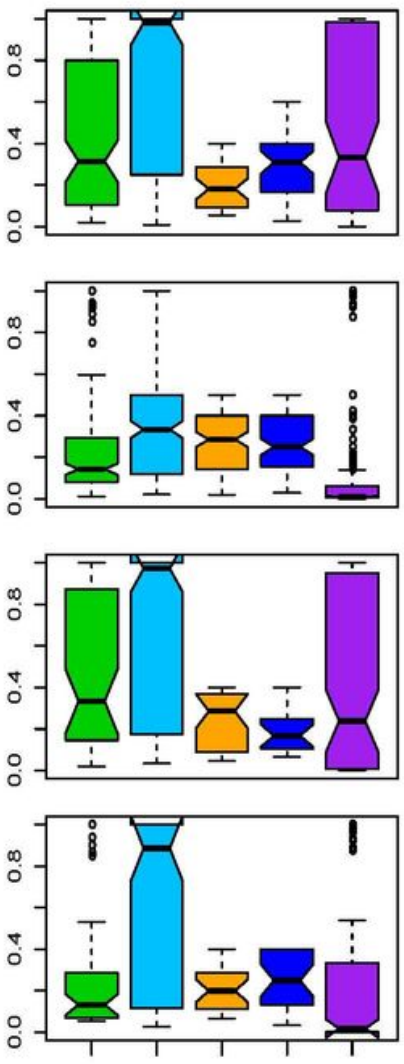

exon
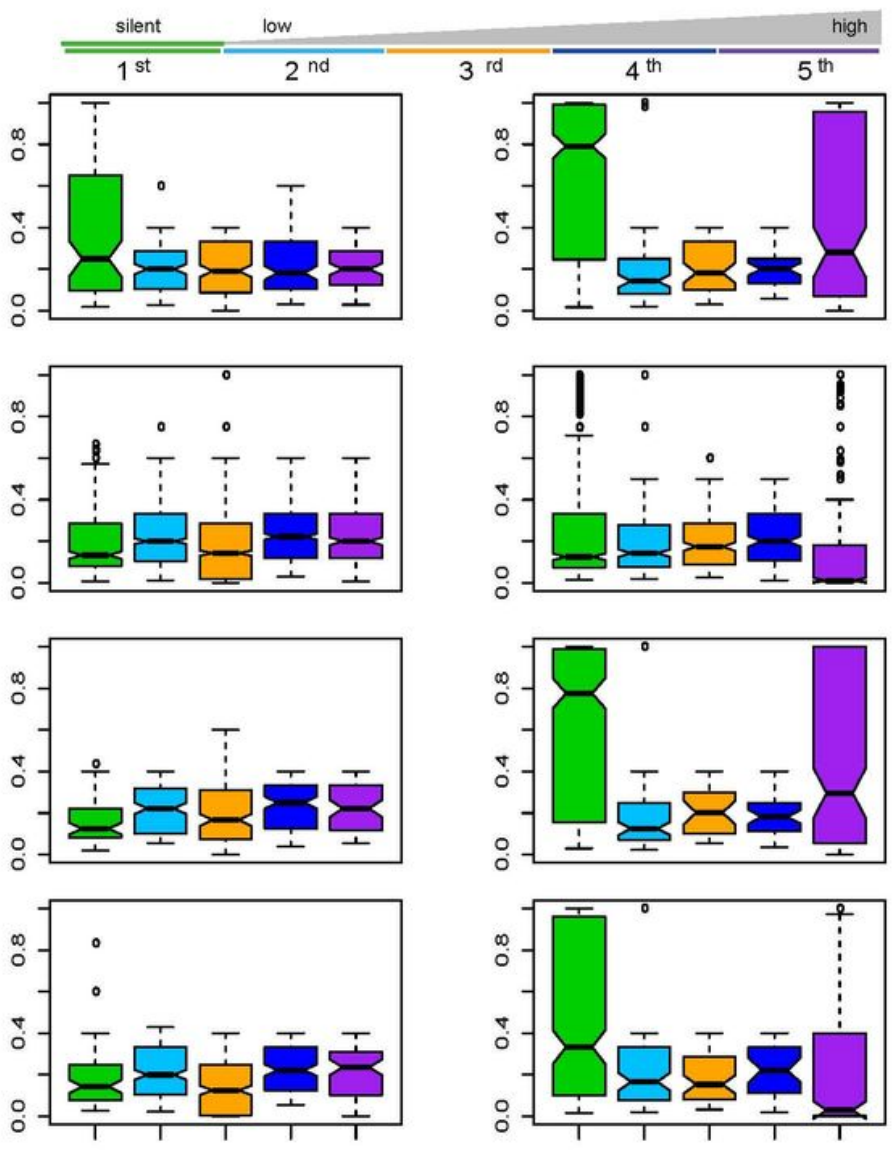

Intron

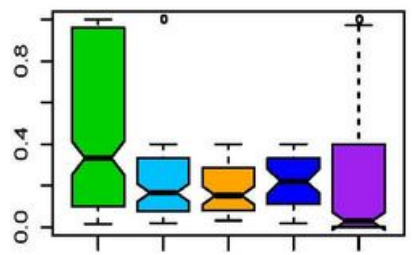

Downstream

Figure 3

Relationship between mC DNA methylation and expression levels of genes in T. solium. Percentage of methylation within genes that were classified based on expression levels. The first class includes silent genes with no sequencing reads detected, and the second to fifth classes cover expressed genes from the lowest $25 \%$ to the highest $25 \%$. Regions of $2 \mathrm{~kb}$ upstream and downstream of each gene was divided into 100-bp intervals, and each gene was divided into 20 intervals ( $5 \%$ per interval). 
A

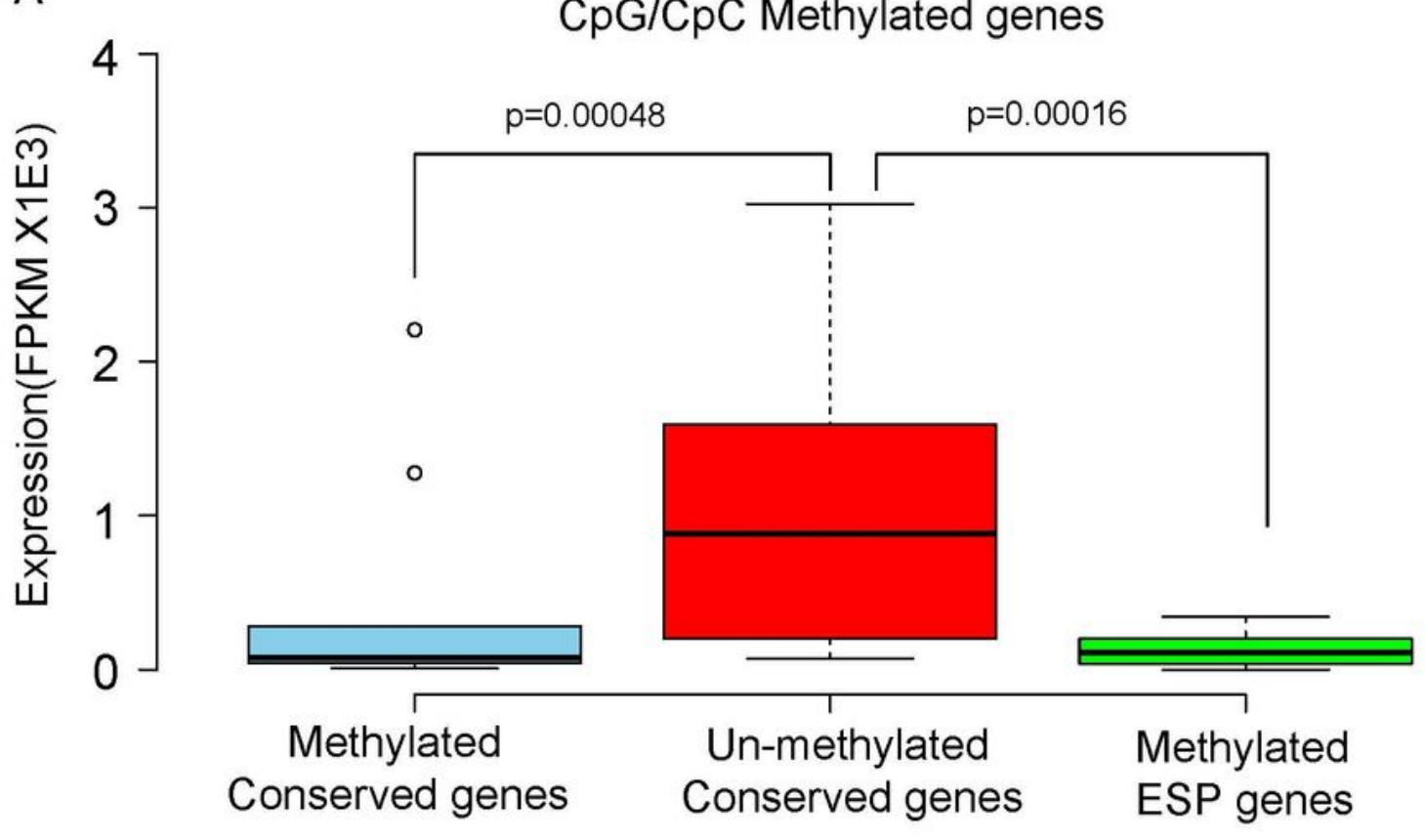

B

$\mathrm{CpA} / \mathrm{CpT}$ Methylated genes

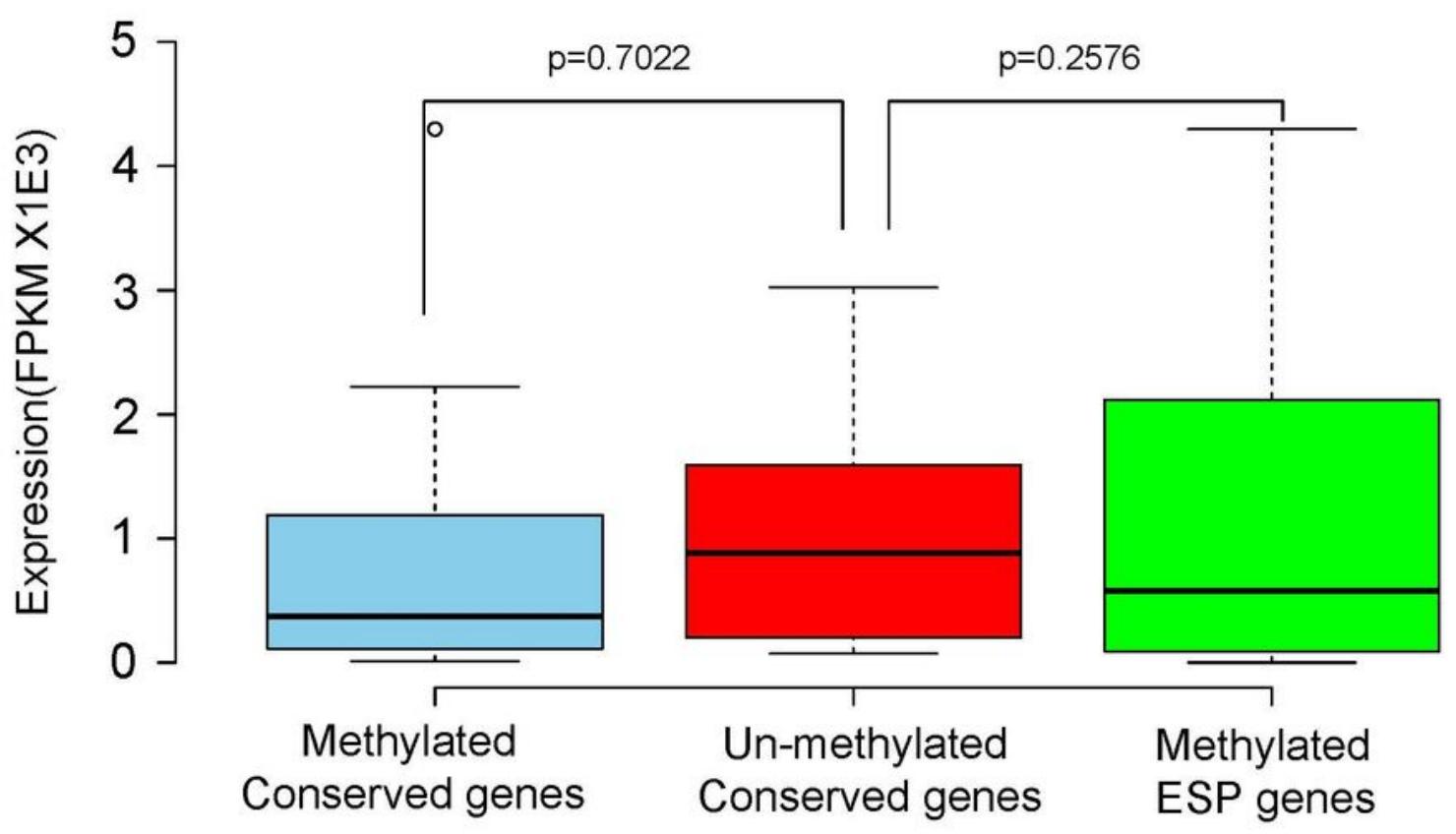

Figure 4

Boxplots of gene expression levels of un-methylated genes, and genes with conserved methylation and methylated ESP genes based on either (A) $\mathrm{CpG} / \mathrm{CpC}$ methylation or (B) $\mathrm{CpA} / \mathrm{CpT}$ methylation. 


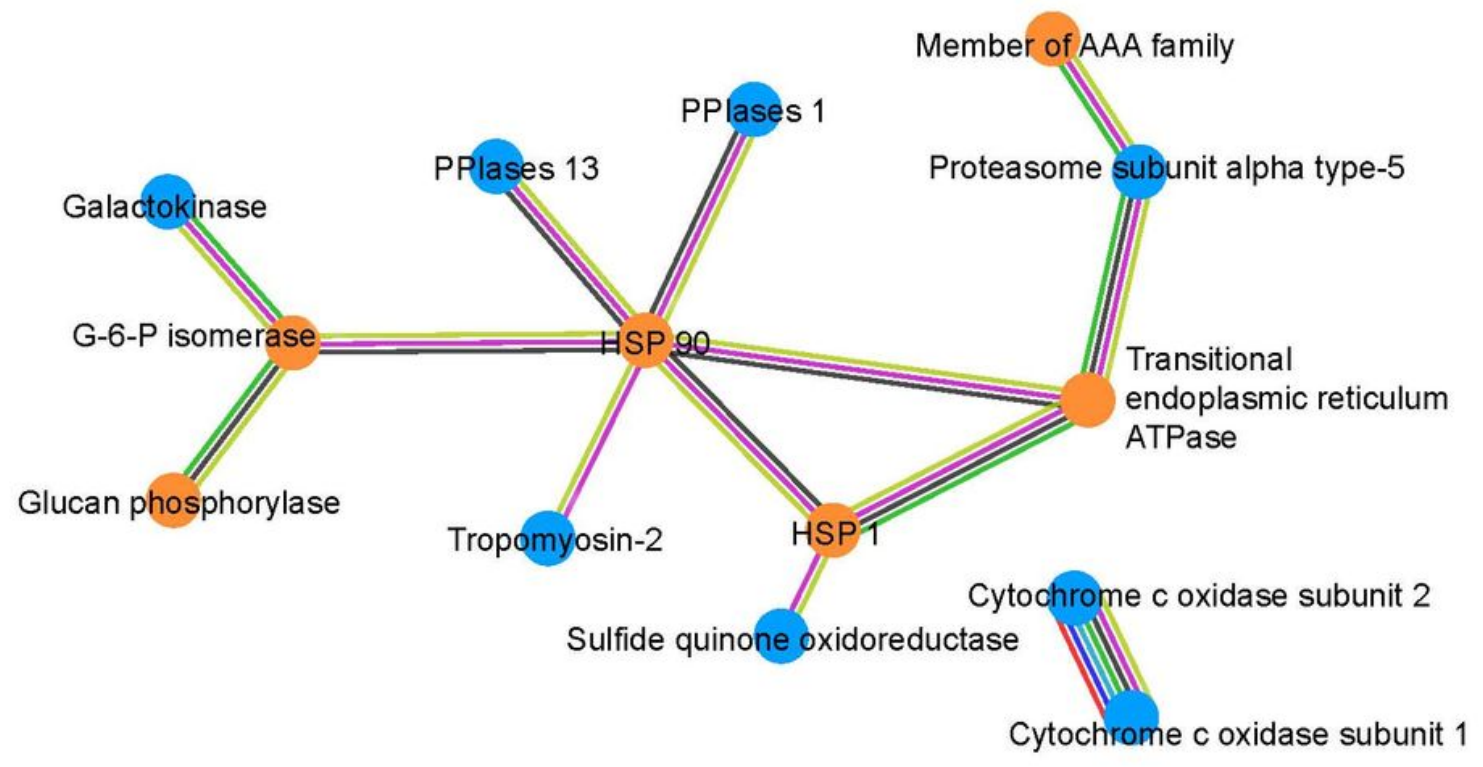

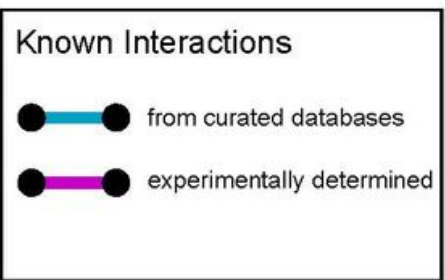

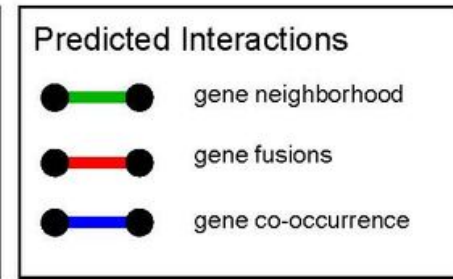

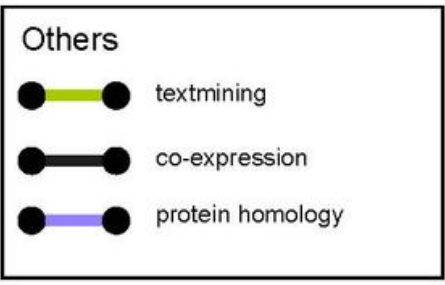

\section{Figure 5}

Protein-protein interactions of conserved genes and ESP genes.

\section{Supplementary Files}

This is a list of supplementary files associated with this preprint. Click to download.

- FigureS5.pdf

- FigureS2.PDF

- FigureS1.pdf

- SupplementalTables.xlsx

- FigureS3.pdf

- FigureS4.pdf

- FigureS6.pdf 